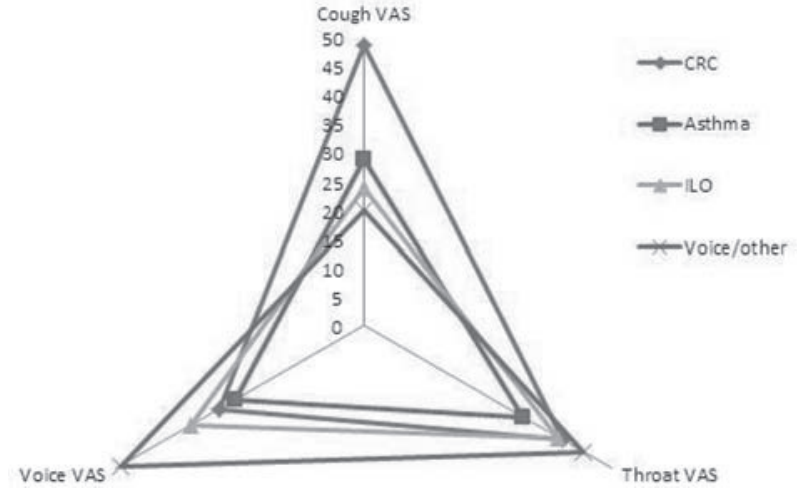

Abstract P109 Figure 1 Mean VAS ratings of cough severity, throat discomfort and voice change according to diagnosis.

\section{P110 FEASIBILITY OF CONTINUOUS LARYNGOSCOPY DURING PROVOCATION IN THE ASSESSMENT OF INDUCIBLE LARYNGEAL OBSTRUCTION}

J Selby, P Cullinan, J Feary, G Scadding, B Fitzgerald, JH Hull. Royal Brompton Hospital, London, UK

\subsection{6/thoraxjnl-2017-210983.252}

Introduction Inducible laryngeal obstruction (ILO) is typically provoked by a range of stimuli, including perfumes and exercise, and characterised by transient throat tightness, dyspnoea and stridor. Central to the diagnosis of ILO is paradoxical adduction of laryngeal structures on inspiration. Continuous laryngoscopy during exercise (CLE) is now established as the gold-standard diagnostic test for exercise-ILO, but diagnosis of other forms of ILO is currently dependent on non-continuous laryngeal visualisation, where crucial diagnostic behaviour of the larynx may be missed. We report the first application of continuous laryngoscopy during provocation (CLP).

Methodology Fifteen female patients (aged 36-58) suspected of having ILO and referred from the RBH specialist upper airway clinic underwent CLP, using self-selected provocation agents to elicit their typical symptoms. Three patients had a prior confirmed diagnosis of asthma. The flexible nasendoscope (CMOS, Karl Storz) was passed without anaesthetic and secured on specialist headgear (figure 1). Patients entered a sealed provocation booth, where they were exposed to the agents they had selected, unable to see the monitor. On elicitation of upper airway symptoms, the specialist SLT initiated laryngeal control strategies with visual biofeedback. The test ended when the patient's typical symptoms had been generated or when exposure exceeded the point of previous symptom provocation.

Results All patients tolerated continuous placement of the scope and confirmed that exposure in the chamber had been sufficiently concentrated to provoke typical symptoms. Only one patient exhibited classical paradoxical glottic movement. Pre-exposure, the vocal cords were closely adducted in three (20\%) patients and fully adducted during unintentional breathholding in three patients (20\%). All patients subsequently attended a review with the specialist SLT to consolidate understanding of CLP findings and use of control strategies.

Conclusion CLP is a safe and well-tolerated method for evaluating laryngeal movement during provocation and negates repeated passage of the nasendoscope. Continuous monitoring throughout provocation testing improves diagnostic accuracy by capturing the presence or absence of paradoxical movement and may be linked with software that tracks laryngeal movement digitally. Further work is needed to develop standardised CLP protocols and identify clinical phenotypes of ILO.

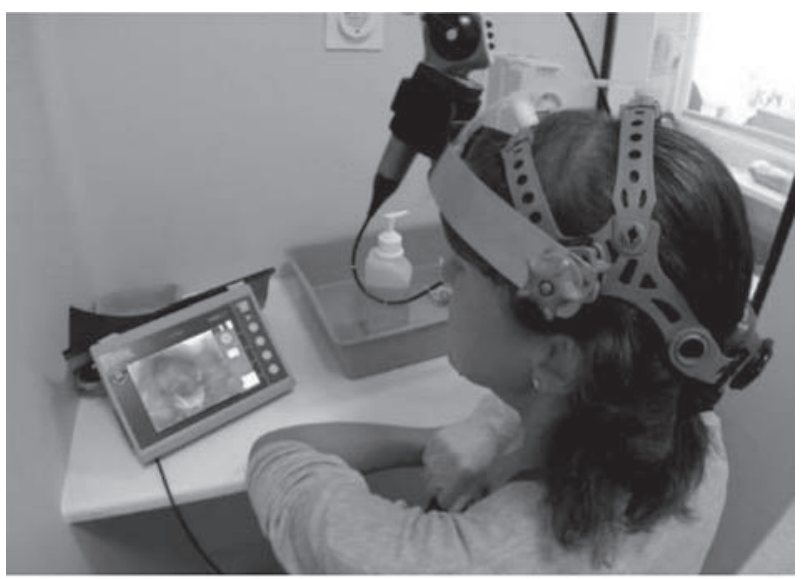

Abstract P110 Figure 1 Demonstration by first author of nasendoscope placement for CLP.

\section{Infected lung: from bench to bedside}

\section{P111 EXHALED BREATH BIOMARKERS IN PULMONARY ASPERGILLOSIS}

${ }^{1}$ ST Talbot, ${ }^{2}$ White, ${ }^{1} \mathrm{~A}$ Hobson, ${ }^{2} \mathrm{M}$ Wilkinson, ${ }^{2} \mathrm{~A}$ Simpson, ${ }^{2} \mathrm{~L}$ Novak-Frazer, ${ }^{2} \mathrm{G}$ GioanTavernier, ${ }^{2} \mathrm{DW}$ Denning, ${ }^{2} \mathrm{~S} J$ Fowler. 'Manchester Medical School, Faculty of Biology, Medicine and Health, The University of Manchester, Manchester, UK; ${ }^{2}$ Division of Infection, Immunity and Respiratory Medicine, School of Biological Sciences, Faculty of Biology, Medicine and Health, Manchester Academic Health Science Centre, The University of Manchester and University Hospital of South Manchester, NHS Foundation Trust, Manchester, UK

\subsection{6/thoraxjnl-2017-210983.253}

Introduction Pulmonary aspergillosis has a substantial global health burden, and is difficult to diagnose and treat. Aspergillus metabolism Results in measurable changes in volatile organic compounds (VOCs) and these may be detectable in the breath. In this pilot study, we sought to measure the abundance of VOCs in the breath samples of patients with pulmonary aspergillosis, and identify any difference in VOC profile between patients with and without Aspergillus in their airway.

Methods Patients were recruited while attending the National Aspergillosis Centre and University Hospital South Manchester, and categorised as Aspergillus positive or Aspergillus negative on the basis of high volume sputum culture. Lower airway breath samples were collected from patients and analysed by thermal desorption gas chromatography mass spectrometry (TD-GC-MS). A target library based upon previous literature reports of Aspergillus-related VOCs was used to deconvolve the raw GC-MS data. Peak area on the chromatogram for each sample was calculated as a measure of VOC abundance. Potentially discriminating compounds between the groups were identified by univariate analysis.

Results 18 patients were recruited, and were allocated to the following groups: culture-positive $(n=7)$, and culture-negative 